\title{
Standard or individualized quality of life for larynx cancer patients?
}

\author{
Roxana Ionescu ${ }^{1}$, Serban V. Bertesteanu ${ }^{1,2}$, Radu C. Popescu ${ }^{1}$, Cristian Balalau ${ }^{1,3}$, \\ Razvan V. Scaunasu ${ }^{1,4}$, Bogdan Popescu ${ }^{1,2}$ \\ ${ }^{1}$ Carol Davila University of Medicine and Pharmacy, Bucharest, Romania \\ ${ }^{2}$ Coltea Clinical Hospital, Department of Otorhinolaryngology, Bucharest, Romania \\ ${ }^{3}$ St. Pantelimon Emergency Hospital, Department of General Surgery, Bucharest, Romania \\ ${ }^{4}$ Coltea Clinical Hospital, Department of General Surgery, Bucharest, Romania
}

\begin{abstract}
Locally advanced larynx cancer benefits from curative surgery therapy. Clinical trials have been proposed for assessing the quality of life for patients with larynx malignant neoplasia who underwent radical surgery. The removal of the "voice box" is known to have great impact on the social reintegration of these patients as well as psychological distress. The presence of tracheostomy and voice impairment are two of the major issues that need to be thought of by patients that will have a total laryngectomy.

The quality of life is a multi-dimensional subjective and personal concept which includes normal physical activity, looks, psychological status, somatic symptoms and, non the less, sexual activity. Cancer free survival intervals for patients with larynx cancer must be evaluated physical and psychological modifications with particular considerations to stress, anxiety and depression related symptoms and to functional rehabilitation adaptation regarding swallowing and speech.

Oncology surgeons must consider that QoL is impaired in all patients with larynx cancer that benefit from total laryngectomy. However, there is evidence presented below that the difference in QoL parameters is small when comparing radical surgery, total laryngectomy, and larynx conservation by radio and chemotherapy.
\end{abstract}

\section{Keywords $\quad$ : larynx cancer, total laryngectomy, QoL, specific questionnaires}

Highlights $\quad \checkmark$ Most parameters of QoL are influenced in larynx cancer patients by the size of tumor and lymph node metastasis.

$\checkmark$ Radical surgery with total laryngectomy presents comparable QoL parameters with radio and chemotherapy conserving the larynx.

To cite this article: Ionescu R, Bertesteanu SV, Popescu RC, Balalau C, Scaunasu RV, Popescu B. Standard or individualized quality of life for larynx cancer patients? J Clin Invest Surg. 2018; 3(2): 62-65. DOI: 10.25083/2559.5555/3.2/62.65 


\section{Introduction}

Locally advanced larynx cancer benefits from curative surgery therapy followed by adjuvant therapy, which will result in the removal of the organ a consecutive permanent tracheostomy and the absence of voice (1). Since these were considered to be the most QoL affecting impairments specific questionnaires aimed swallowing disorders and aphonia, mostly (2). Clinical trials have been proposed for assessing the quality of life for patients with larynx malignant neoplasia who underwent radical surgery. The removal of the 'voice box' is known to have great impact on the social reintegration of these patients as well as psychological distress.

The presence of tracheostomy and voice impairment are two of the major issues that need to be thought of by patients that will have a total laryngectomy. Some patients refuse surgery due to the lack of voice communication considering the impairment too hard to be accepted, and in some cases embarrassing in relation to their family and friends. Ethnic considerations are to be taken into consideration as some ethnic groups outcast their members if such an invalidity occurs. It is considered that the evaluation of the patients needs and satisfaction regarding the daily living functionality in performing different types of activities is mandatory for patients with total laryngectomy. These patients need to feel that the ideal of a normal-like living is possible.

The normality comes from the ability of communicating with fellow individuals, normal oral food and beverage intake and the feeling of noninvalidity. Quality of life personal perspective can be viewed as an individual perception of life course. The prognosis of the malignant disease as well as the prognosis of the oncology therapy is of outmost importance for the patient since, although clinically valid arguments support the idea of survival for as long as possible, most of the patients place into balance possible complications with the lack of therapy, or more often less invasive oncology therapy.

The preservation of the organ, the larynx, is preferred in patients with high cognitive output, active individuals and in patients with a poor psychological status. The landmark for cancer patients is the predisease daily status concerning all the activities. Therefore, there is a need to tailor the goals of the oncology therapy to meet the expectations of the patient regarding previous life-style standard.

\section{Discussions}

World Health Organization has a special division that analyses data from different research centers to better understand the quality of life issue for different types of pathology. However, this is a subjective perspective since patients have their own way of perceiving the problem.

\section{QoL concept for larynx cancer patients}

The quality of life is a multi-dimensional subjective and personal concept which includes normal physical activity, looks, psychological status, somatic symptoms and, non the less, sexual activity (3). Physical activity for patients with total laryngectomy is dependent on the general status of the patient and on the patency of the tracheostomy.

In general, patients that undergo adjuvant oncology therapy, such as radiation therapy, may experience some difficulties in breathing due to the chronic inflammation of the respiratory mucosa which is unable to modify the parameters of inhaled air in terms of humidity, purity and temperature. This can cause the patient to cough and to reduce the tolerance to effort. Cancer patients are considered to have a handicap in terms of physical activity in such ways that they are not supposed to perform heavy duty activities. In women with this pathology the effect of oncology therapy is less intense than in men, who are used to perform heavier physical activities. Men patients need to adapt their life style in terms of physical activity intensity, which can cause distress in their psychological status. Psychological therapy may be needed for these patients (4).

Considering that these patients undergo surgery that cause scaring, physical aspect is very important in some cases. If there is the case of multiple and series of surgery the overall look of the patient is to be severely affected. In the pre-therapy discussion with the patient there is the need for informed consent also from the perspective of visual aspect of the cervical region. There are patients, especially high-class members, that are particularly interested in the physical outcome of surgery and, eventually, radiation therapy. However, physical aspect, although important it is not a major cause of therapy rejection. Secondary effects of external beam radiotherapy and the loss of hair after chemotherapy may cause distress to the patient. Before the beginning of therapy these situations need to be presented to the patient and physicians need to have a written consent of the patient (5). 
Somatic symptoms such as pain, paresthesia, dehydration, cachexia, nausea, vomiting. Symptoms are cognitive and functional impairment are to be thought in relation to the affected organ or system during off, communicated to the patient and addressed if it oncology therapy. occurs. Pain is related to the complications of the oncology therapy, mainly due to radiotherapy, since surgery related pain is limited in time. There are situations in which scaring tissue leads to nerve traction with concurrent pain and referred pain. Oral surgery and extended laryngectomy may lead to functional impairment in the cervical area, with the possibility of decreased mobility or the inability of normal mouth opening with limited oral food and beverage intake.

Therapy response must be evaluated based on the severity of the disease in direct relation to the physiological needs of the patient who must bare the stress caused by both the disease and the oncology therapy. Functional stress is determined by using the Karnofksy index. Elderly patients and those with a lower value of the index have a poorer prognosis. This, however, is debatable at least. The appreciation of the performance status is done by using both the Karnofksy index and the ECOG index. Both tools are to be used when dealing with a malignancy, especially in the head and neck region due to the breathing and feeding issues. For each individual patient the physician needs to evaluate whether the patient is capable to support daily activities on its own or the patient needs to be assisted. The quality of life is improved in patients for whom normal activities are performed without any assistance, whether they are at home or at work. Social reintegration is wanted for laryngectomee patients with the upside that they feel useful, have a purpose and start new projects in their life. On the other hand, anxiety and depression may occur if daily situations are being performed in an assisted manner. This is the case of home medical assistance for patients that have a degree of invalidity. Psychological support is mandatory in these situations as well as the efforts of family and friends (6).

\section{Paliative care}

In the case of locally advanced disease or in the case of distant site metastasis paliative therapy is the only means of medical care available. This type of therapy comprises local wound care, permanent, active and continuous. The goal of the paliative care is to reduce pain, to ensure normal-like feeding and to address oncology related symptoms. These symptoms are halitosis, dry mouth, stomatitis, oral candidosis, taste impairment, anorexia, dysphagia, odynophagia,

\section{Nutrition therapy}

Clinical nutrition represents the means of administering nutrients to cancer patients which influences the prognosis, quality of life and survival rates. Nutrition is an important part of the oncology therapy this meaning that this is a team effort. Nutritional status is defined by the balance between intake and consumption. It is well known that malignancies are consumptive, therefor the oncology therapy team needs to establish which patients are at risk and immediately start nutrition therapy (4). According to the European Society of Enteral and Parenteral Nutrition (ESPEN) cancer patients need to receive nutrients 1.3 times normal values if they have a normal body mass index and 1.7 times normal values if the patients are malnourished. Serum albumin needs to be evaluated since healing process and immunological response are directly connected. Some 60 to $80 \%$ of the larynx cancer patients with advanced local disease are diagnosed as cachectic at hospital admission. Nutritional status evaluation needs to be performed as soon as possible and nutrition therapy needs to be started according to the data gathered by specific scores, such as NRS 2002. If feeding via the digestive tube is preferred if the digestive system is functional. If not, parenteral or mixed nutrition are to be used.

According to a study performed by Villaseca et al. long-term QoL parameters in patients that underwent total laryngectomy is at similar levels with normal population. This somewhat of a paradox may be justified by the fact that the tools used to assess physiological, nutritional and psychological status were general health instruments. Larynx cancer specific QoL questionnaires showed that decreased parameters are present in these patients. Some other categories described in QoL questionnaires were directly correlated with modifications in the subscales of prediction, such as age, sex, pre-oncology psychological status and type of adjuvant therapy (5). To assess the parameters of QoL for larynx cancer patients physicians must use different types of tools, general and specific (6).

QoL has been evaluated in a series of studies regarding the size of the tumor and the local metastasis. The conclusion was that most parameters of QoL are being influenced by the $\mathrm{T}$ (size of tumor) and $\mathrm{N}$ (lymph node metastasis). This is the case in which total 
laryngectomy is performed in an extended oncology surgery. Otherwise, if the tumor does not extend over the limit of the larynx, total laryngectomy has the same outcome in QoL questionnaires $(7,8)$. However, there is a difference in psychological status and overall functionality in direct correlation with time lapsed from the beginning of oncology therapy and the end of all rehabilitation procedures. Along with this the type of adjuvant therapy in linked with modified QoL status (9).

\section{Conclusions}

Cancer free survival intervals for patients with larynx cancer must be evaluated physical and psychological modifications with particular considerations to stress, anxiety and depression related symptoms and to functional rehabilitation adaptation regarding swallowing and speech. Oncology surgeons must consider that QoL is impaired in all patients with larynx cancer that benefit from total laryngectomy. However, there is evidence, as presented above, that the difference in QoL parameters is small when comparing radical surgery, total laryngectomy, and larynx conservation by radio and chemotherapy.

\section{Conflict of interest disclosure}

There are no known conflicts of interest in the publication of this article, and there was no financial support that could have influenced the outcomes. The manuscript was read and approved by all authors.

\section{References}

1. Guibert M, Lepage B, Woisard V, Rives M, Serrano E, Vergez S. Quality of life in patients treated for advanced hypopharyngeal or laryngeal cancer. Eur Ann Otorhinolaryngol Head Neck Dis. 2011; 128(5): 218-23.

2. Fung K, Lyden TH, Lee J, Urba SG, Worden F, Eisbruch A, Tsien C, Bradford CR, Chepeha DB, Hogikyan ND, Prince ME, Teknos TN, Wolf GT. Voice and swallowing outcomes of an organpreservation trial for advanced laryngeal cancer. Int
J Radiat Oncol Biol Phys. 2005; 63(5): 1395-9. PMID: 16087298

DOI: 10.1016/j.ijrobp.2005.05.004

3. Ramírez MJ, Ferriol EE, Doménech FG, Llatas MC, Suárez-Varela MM, Martínez RL. Psychosocial adjustment in patient surgically treated for laryngeal cancer. Otolaryngol Head Neck Surg. 2003; 129(1): 92-7. PMID: 12869923, DOI: 10.1016/S0194-59980300478-9

4. Riboli E, Kaaks R, Estève J. Nutrition and laryngeal cancer. Cancer Causes Control. 1996; 7(1): 147-56. PMID: 8850442

5. Vilaseca I, Chen AY, Backscheider AG. Long-term quality of life after total laryngectomy. Head Neck. 2006; 28(4): 313-20. PMID: 16200627, DOI: 10.1002/hed.20268

6. Hanna E, Sherman A, Cash D, Adams D, Vural E, Fan CY, Suen JY. Quality of life for patients following total laryngectomy vs chemoradiation for laryngeal preservation. Arch Otolaryngol Head Neck Surg. 2004; 130(7): 875-9. PMID: 15262766, DOI: 10.1001/archotol.130.7.875

7. Morton RP. Studies in the quality of life of head and neck cancer patients: results of a two-year longitudinal study and a comparative crosssectional cross-cultural survey. Laryngoscope. 2003; 113(7): 1091-103. PMID: 12838004, DOI: 10.1097/00005537-200307000-00001

8. Bjordal K, Ahlner-Elmqvist M, Hammerlid E, Boysen M, Evensen JF, Biörklund A, Jannert M, Westin T, Kaasa S. A prospective study of quality of life in head and neck cancer patients. Part II: Longitudinal data. Laryngoscope. 2001; 111(8): 1440-52. PMID: 11568582

DOI: $10.1097 / 00005537-200104000-00021$

9. Kazi R, Prasad V, Venkitaraman R, Nutting CM, Clarke P, Rhys-Evans P, Harrington KJ. Questionnaire analysis of the swallowing-related outcomes following total laryngectomy. Clin Otolaryngol. 2006; 31(6): 525-30. PMID: 17184459, DOI: 10.1111/j.1365-2273.2006.01335.x 